\section{Case report 662}

\section{M.A. Nuovo, H.A. Sissons, J.D. Zuckerman}

Skeletal Radiology (1991) 20:217-221

In Case report 662 (Diagnosis: Bilateral avascular necrosis of femur, with supervening suppurative arthritis of right hip) the first four paragraphs of the Discussion (p. 220) were omitted. The section should begin as follows:

\section{Discussion}

Suppurative arthritis is known to involve a wide range of joints in individuals of all ages, and in a variety of circumstances. Many different organisms have been implicated $[6,22]$. A focus of infection elsewhere in the body often precedes the joint infection, which is presumed to develop as a result of bacteremia or septicemia.

General conditions that predispose to the development of suppurative arthritis include complement deficiencies, disorders of chemotaxis, impaired host defense mechanisms including the effects of immunosuppressive drugs and glucocorticosteroids, hypogammaglobulinemia and diabetes mellitus [6].

In recent years, intravenous drug abuse has come to be seen as an important background for the development of suppurative arthritis [1, 17, 25]. While drug abuse is clearly a factor in the case we report, most examples of suppurative arthritis in drug abusers, unlike the present case, involve the joints of the vertebral column and the fibrocartilaginous articulations of the pelvis and sternum. Involvement of major diarthrodial joints is relatively infrequent. While a variety of infecting organisms have been reported, many cases are due to Pseudomonas aeruginosa, rather than to Staphylococcus aureus or the other organisms commonly involved in the absence of drug abuse.

A considerable number of joint diseases have been reported as predisposing factors for suppurative arthritis. Outstanding among these is rheumatoid arthritis, where the development of bacterial joint infection is recognized as an important and not infrequent complication $[5,12,13,20,21]$. Osteoarthritis [6], Charcot's arthropathy $[15,26]$, systemic lupus erythematosus $[10$, 24], and various types of crystal induced arthritis [2, $9,14]$ have also been reported, although less frequently, in association with suppurative arthritis. 